\title{
Synthesis and Spectroscopic Characterization of Undoped Nanocrytalline ZnO Particles Prepared by Co-Precipitation
}

\author{
Suhendro Purbo Prakoso, Rosari Saleh * \\ Departemen Fisika, FMIPA-Universitas Indonesia, Depok, Indonesia. \\ Email: *rosari.saleh@ui.ac.id \\ Received May $8^{\text {th }}, 2012$; revised June $9^{\text {th }}, 2012$; accepted July $1^{\text {st }}, 2012$
}

\begin{abstract}
A series of undoped nanocrystalline $\mathrm{ZnO}$ particles were successfully synthesized at various dry temperatures $\left(100^{\circ} \mathrm{C}\right.$ $600^{\circ} \mathrm{C}$ ) using coprecipitation method. The samples were characterized using a variety of experimental methods such as $\mathrm{x}$-ray diffraction (XRD), energy dispersive x-ray spectroscopy (EDX), thermal analysis TG-DTA, UV-vis spectroscopy, infrared absorption spectroscopy (FTIR) and electron spin resonance spectroscopy (ESR). According to XRD analysis, all of our $\mathrm{ZnO}$ samples posses the hexagonal wurzite structure with average crystallite size increased ranging from 19 $23 \mathrm{~nm}$ as dry temperature increased. Optical absorption spectra show that the band gap shifted to the lower energy with increasing crystallite size. ESR measurements showed the resonance of electron centers with the g values of about 1.96. With increasing dry temperature we observed the decrease of the $g$ values and the increase of the intensities of the ESR signal. In addition an increase in dry temperature results in a pronounce decrease of $\mathrm{OH}$ local vibrational modes. The results from ESR measurements are well supported by the results obtained from Infrared absorption spectroscopy and thermal analysis measurements.
\end{abstract}

Keywords: ZnO Nanoparticles; Structural and Optical Properties; Magnetic Resonance

\section{Introduction}

Although the interest of research in semiconductor $\mathrm{ZnO}$ has started in 1935 [1], it was first in 1950s did it gain more credence mainly due to its optical properties [2]. In many respects $\mathrm{ZnO}$ stands somewhat between the heteropolar alkali halides and the homopolar semiconductors group IV elements, and it is often considered as an electronic semiconductor with a large band gap. Thus, replacement of zinc atoms by an element of group I-A or I-B or oxygen atoms by an element group V-A, should result in the formation of acceptor centers, while replacement of zinc atoms by an element of group III-A or oxygen atoms by an element group VII-A, should lead to the formation of donor centers [3] as well as zinc and oxygen vacancies could act as acceptor and donor, respectively. Recently, it was shown that $\mathrm{ZnO}$ possess superior properties such as a high exciton binding energy combined with a low lasing threshold density and a good resistance to bombardment with high energy particles [4]. It is one of the promising candidates as a serious alternative to GaN for use in optoelectronic devices and UV

${ }^{*}$ Corresponding author. lasers operating at high temperatures and harsh environments. However, almost all optoelectronic applications require the control of conductivity from n-type to p-type. One of the major obstacles that have to be overcome to allow for successful development of optoelectronic devices and lasers is the difficulty in finding an efficient p-type dopant.

As grown, $\mathrm{ZnO}$ is almost always exhibits $\mathrm{n}$ type conductivity, with electrons in the conduction band as the charge carriers. The origin of this conductivity has been discussed for years and is still controversial. Traditionally, the nature of this conductivity has been attributed to native defects. However, Van de Walle [5] in his firstprinciples investigation, based on density functional theory suggested that the presence of hydrogen atom in $\mathrm{ZnO}$ can act as a shallow donor. This behavior is unexpected and very different from hydrogen's role in other semiconductors, in which it acts only as a compensating center counteracting the prevailing conductivity. The presence of hydrogen in the crystal-growth is not surprising and it is very difficult to avoid its incorporation into the sample during the process of the crystal-growth.

In nanoscale semiconductor materials, $\mathrm{ZnO}$ is of ex- 
traordinary importance and a substantial number of papers have been published reporting on $\mathrm{ZnO}$ nanostructure/nanoparticles. As the material becomes smaller it possess additional properties, namely 1) the quantumsize effect, that influences the bandgapsize [6,7], and consequently the optical and electronical properties of the materials, and 2) a large ratio of surface to volume relative to bulk materials. The latter property is relevant to hydrogen storage and catalysis. Several synthesis methods of $\mathrm{ZnO}$ nano particles have been reported in the past years, such as sol-gel chemistry [8], sonochemical method [9], hydrothermal method [10], combustion method [11] and coprecipitation method [7]. Controlling the synthesis condition is a critical point, because the properties of the nanoparticles are found to vary with synthesis methods. The variation in optical and electronical properties is assigned to different parameter such as $\mathrm{pH}$, aging time, reaction temperature as well as dry temperatures.

In this article we report the synthesis of nanocrystalline $\mathrm{ZnO}$ particles obtained using coprecipitation method at various dry temperature and characterize the structural, optical properties, vibrational modes and magnetic resonance of these nanoparticles. Structure is characterized using x-ray diffraction (XRD), optical properties and vibrational modes are studied by UV-vis and infrared absorption spectroscopy, while magnetic resonance are deployed using electron spin resonance (ESR) spectroscopy. Through these measurements we attempt to correlate one property to the others. Our results are compared with those published in the literatures.

\section{Experimental}

For the synthesis of undoped $\mathrm{ZnO}$ nanoparticles in this study, the zinc sulfate hepta hydrate $(99.9 \%) \mathrm{ZnSO}_{4} \cdot 7 \mathrm{H}_{2} \mathrm{O}$, $25 \%$ aqueous $\mathrm{NaOH}$ and ethanol were used which are procured from Aldrich. All of the chemicals used were analytical reagent grade and were directly used without further purification. Synthesis of $\mathrm{ZnO}$ nanoparticles was carried out using coprecipitation method. $24.6 \mathrm{mmol}$ analytical grade of $\mathrm{ZnSO}_{4} \cdot 7 \mathrm{H}_{2} \mathrm{O}$ from Aldrich was dissolved in $250 \mathrm{ml}$ de-ionized water with vigorous stirring at temperature of $80^{\circ} \mathrm{C}$. Simultaneously, appropriate amount of $\mathrm{NaOH}$ was dissolved in $500 \mathrm{ml}$ of de-ionized water. Then, both solutions were mixed with constant stirring at temperature of $80^{\circ} \mathrm{C}$ for 30 min until a milky white solution was obtained. After the reaction process, the solution was centrifuged at $3000 \mathrm{rpm}$ for $10 \mathrm{~min}$ and washed for several times with deionized water and ethanol to remove the by-product sodium sulfate $\left(\mathrm{NaSO}_{4}\right)$. The precipitate was cooled and aged at room temperature for $24 \mathrm{~h}$. To obtain various concentration of hydrogen the precipitate was dried in a vacuum oven at various dry temperatures, $\mathrm{Td}\left(100^{\circ} \mathrm{C}-600^{\circ} \mathrm{C}\right)$ for $4 \mathrm{~h}$. Some samples were annealed at annealing temperature up to $800^{\circ} \mathrm{C}$ for 6 h.

The structural characterization has been carried out by using a standard x-ray diffractometer (Philips PW1710) with monochromatic $\mathrm{Cu}-\mathrm{K} \alpha(\lambda=1.54060 \AA)$ radiation operated at $40 \mathrm{kV}$ and $20 \mathrm{~mA}$ in the range from $10^{\circ}$ to $80^{\circ}$. The calibration of the diffractometer was done using Si powder. The XRD patterns of the specimens were verified by comparison with the JCPDS data. The average size of precipitate crystallites $\langle\mathrm{D}>$ was estimated by using the Scherrer peak broadening method: $\langle\mathrm{D}\rangle=k \lambda / \Delta$ $\cos \Theta$, where $k=$ shape factor $(0.89), \lambda$ is $\mathrm{x}$-ray wavelength, $\Delta$ is line broadening at half-height and $\Theta$ is Bragg angle of the particles. The average crystallites size is obtained from the most intense peak, corresponding to (101) reflection by using the Debye-Scherrer formula. Elemental analysis of the samples have been done by energy dispersive x-ray spectroscopy (EDX) using scanning microscope. Optical characterizations were carried out by measuring the diffuse reflectance spectroscopy. All spectra were taken in the range of $200-800 \mathrm{~nm}$ using shimadzu UV-vis spectrophotometer with integrating sphere attachment and spectralon reflectance standard. In order to study the bonding configuration infrared absorption measurements were performed in the spectral range of 400 to $4000 \mathrm{~cm}^{-1}$ using a Shimadzu Fourier transform spectrometer. Pellet made with $\mathrm{KBr}$ powder and nanoparticles was used for the infrared absorption studies. To obtain information on defects and vacancies electron spin resonance (ESR) was carried out using X-band JEOL JES-RE1X at room temperature. The shape and the area of the ESR spectra were analyzed by standard numerical methods. Simultaneous thermogravimetric and differential thermal analysis (TGA-DTA) measurements were performed using Setaram TAG 24. The samples were heated from room temperature up to $800^{\circ} \mathrm{C}$ at $10^{\circ} \mathrm{C} \cdot \mathrm{min}^{-1}$.

\section{Results and Discussion}

Figure 1(a) shows the XRD patterns of samples synthesized by coprecipitation methods with dry temperature $\mathrm{Td}$ of $100^{\circ} \mathrm{C}, 200^{\circ} \mathrm{C}, 400^{\circ} \mathrm{C}$ and $600^{\circ} \mathrm{C}$ for $4 \mathrm{~h}$. The spectra are almost equal to the typical XRD spectra of $\mathrm{ZnO}$ nanoparticles reported from other experiments $[12$, 13]. In all our XRD patterns nine peaks are observed around $2 \Theta=32.12,34.48,36.6,47.76,56.84,63.02$, $66.95,68.23,69.24$ which correspond to (100), (002), (101), (102), (110), (103), (200), (112) and (201), respectively. For each sample, all observed peaks can be indexed as the hexagonal wurzite structure of $\mathrm{ZnO}$ with having space group $\mathrm{P}_{63} m c$. The existence of any impurity compound other than wurzite structure is not observed.

These results are consistent with the results obtained 
from EDX spectra in Figure 1(b). In addition to an Oxygen peak at $0.6 \mathrm{keV}, \mathrm{Zn}$ signals at about 1.01, 8.7 and 9.5 $\mathrm{keV}$ was observed, indicative of successful synthesis of $\mathrm{ZnO}$ nanoparticles as already confirmed by XRD data.

The values of lattice parameters calculated from XRD data by using the Rietveld refinement analysis are shown in Figures 2(a) and (b), respectively. All available reflections were fitted with the Gaussian distribution. Analysis of XRD patterns Figure 1(a) suggested that the lattice parameters for $\mathrm{ZnO}$ obtained with dry temperature $T d=100^{\circ} \mathrm{C}$ are $a=b=3.255 \AA$ and $c=5.218 \AA$. There is no significant difference in calculated lattice parameters as dry temperature is increased from $100^{\circ} \mathrm{C}$ to $600^{\circ} \mathrm{C}$. These results are slightly higher than the standard JCPDS parameter for bulk $\mathrm{ZnO}, a=b=3.2498 \AA$ and $c=5.206$ $\AA$.

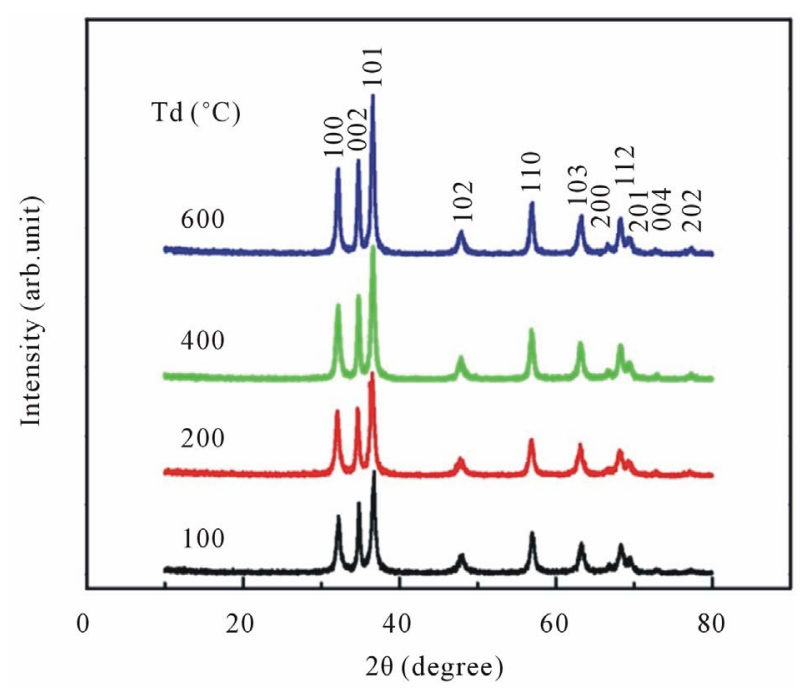

(a)

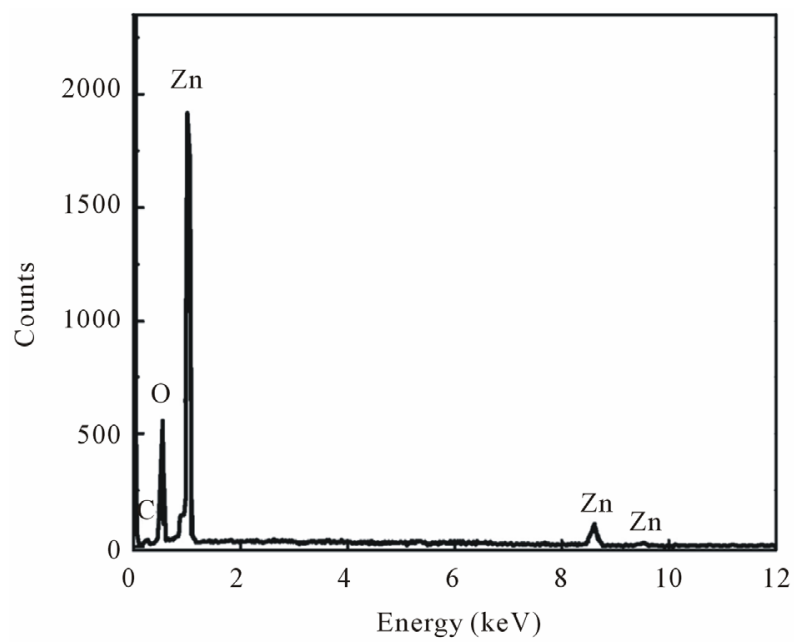

(b)

Figure 1. (a) X-ray diffraction patterns of $\mathrm{ZnO}$ nanoparticles synthesized with different dry temperatures; (b) EDX spectra of $\mathrm{ZnO}$ nanoparticles.

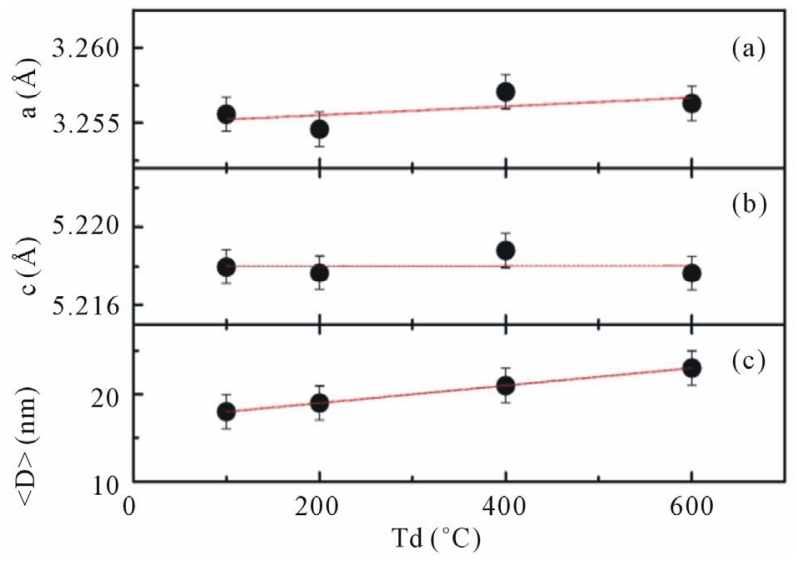

Figure 2. Panel (a) and (b) show lattice parameters a and c of hexagonal wurzite $\mathrm{ZnO}$ calculated using the (101) and (002) peaks as a function of dry temperature; Panel (c) shows the average crystallite size obtained from Scherrer's formula. Lines are guides to the eye.

Moreover, a careful analysis of peak positions suggestive a small shifting in its value toward a lower $2 \Theta$ with increasing dry temperature, indicating a presence of compressive strain in the samples [14]. It is also shown that for the entire samples the reflection peaks become sharper and the full width at half maximum (FWHM) are slightly decreased with increasing dry temperature.

Using the Scherrer peak broadening method, the average crystallite size obtained are $\sim 18 \mathrm{~nm}$ for $T d$ of $100^{\circ} \mathrm{C}, \sim 19 \mathrm{~nm}$ for $T d$ of $200^{\circ} \mathrm{C}, \sim 21 \mathrm{~nm}$ for $T d$ of $400^{\circ} \mathrm{C}$ and $\sim 23 \mathrm{~nm}$ for $T d$ of $600^{\circ} \mathrm{C}$ (Figure 2(c)), thus indicating a little enhancement in average crystallite size at higher dry temperature.

In order to reveal the changes that occured during thermal treatment of the precursor, TG-DTA analysis were carried out from room temperature to $600^{\circ} \mathrm{C}$ in atmosphere (see Figure 3). According to the TG curve the precursor loses its weight in two major steps.

The first major step is in the temperature range below $100^{\circ} \mathrm{C}$ and indicated a loss of $3.2 \mathrm{wt} \%$ revealing the dehydrogenation process of surface-adsorbed water molecules.

The second step of weight loss $(2.6 \mathrm{wt} \%)$ appeared in the range of $100^{\circ} \mathrm{C}-220^{\circ} \mathrm{C}$ could be associated with out diffusion of $\mathrm{OH}^{-}$ions or hydrogen atoms from the $\mathrm{ZnO}$ network. A further out diffusion of $\mathrm{OH}^{-}$ions or $\mathrm{H}$ atoms is observed from a slightly decrease of the TG curve at temperatures higher than $220^{\circ} \mathrm{C}$. Since the total weight loss in the entire thermal analysis study was less than 8 $\mathrm{wt} \%$ indicating the major amount of $\mathrm{ZnO}$ formation during synthesis. The DTA curve of the precursor obtained during thermal treatment exhibits two endothermic peaks correspond to two major weight loss steps of TGA curve.

Optical characterization was carried out by measuring 


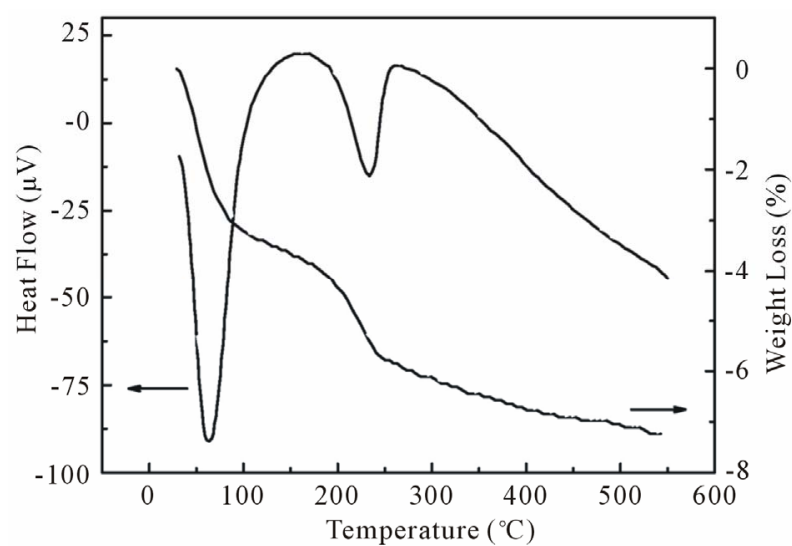

Figure 3. TG curve showing the weight loss for $\mathrm{ZnO}$ nanoparticles as a function of temperature and its corresponding DTA curve.

the diffuse reflectance spectroscopy. All spectra were taken in the range of $200-800 \mathrm{~nm}$. Figure 4 shows the diffuse reflectance spectra $\mathrm{R}$ as a function of wavelength for samples shown in Figure 1(a). Since our samples are powder, the low reflectance values in our spectra indicate high absorption in the corresponding wavelength region. The data collected at room temperature showed a clear difference between the samples in the region of $350-800$ $\mathrm{nm}$. The significance of this is more visible after applying the Kubelka-Munk function $F(R)$ given by the relation $F(R)=(1-R)^{2} / 2 R$, where $R$ is the magnitude of reflectance [15]. The optical gap (inset of Figure 4) was estimated from diffuse reflectance spectra by plotting the square of the Kubelka-Munk function $F(R)^{2}$ as a function of energy. To obtained the optical gap the linear part of $F(R)^{2}$ curve was extrapolated until it intersects the energy axis at $F(R)^{2}=0$.

The optical gap of $\mathrm{ZnO}$ dried at $100^{\circ} \mathrm{C}$ determined by the above method to be $3.52 \mathrm{eV}$, which is higher than that for bulk $\mathrm{ZnO}$ (3.32 eV). The corresponding optical gaps are seen to be shifted to lower energies with increasing dry temperature. The optical gap of our $\mathrm{ZnO}$ samples was found around $3.52 \mathrm{eV}$ for $\mathrm{Td}$ of $100^{\circ} \mathrm{C}, 3.46$ $\mathrm{eV}$ for $T d$ of $200^{\circ} \mathrm{C}, 3.38 \mathrm{eV}$ for $\mathrm{Td}$ of $400^{\circ} \mathrm{C}$ and $3.35 \mathrm{eV}$ for $T d$ of $600^{\circ} \mathrm{C}$. The variation of optical gap in $\mathrm{ZnO}$ has been observed by several authors $[12,16]$, who is explained that the change in optical gap on the basis of variation of the average particle size.

A typical ESR spectrum of $\mathrm{ZnO}$ dried at $T d=200,400$ and $600^{\circ} \mathrm{C}$ are shown in Figures 5(a)-(c), respectively. Almost symmetric resonance was observed, and the resonance shifts to a higher field, which corresponds to the decrease in $g$-value. The quenching of the linewidth can be seen as the dry temperature is increased. The intensity, on the other hand increased with increasing dry temperature. The ESR signal in the sample dried with $T d=$ $100^{\circ} \mathrm{C}$ was found very weak compared to samples dried

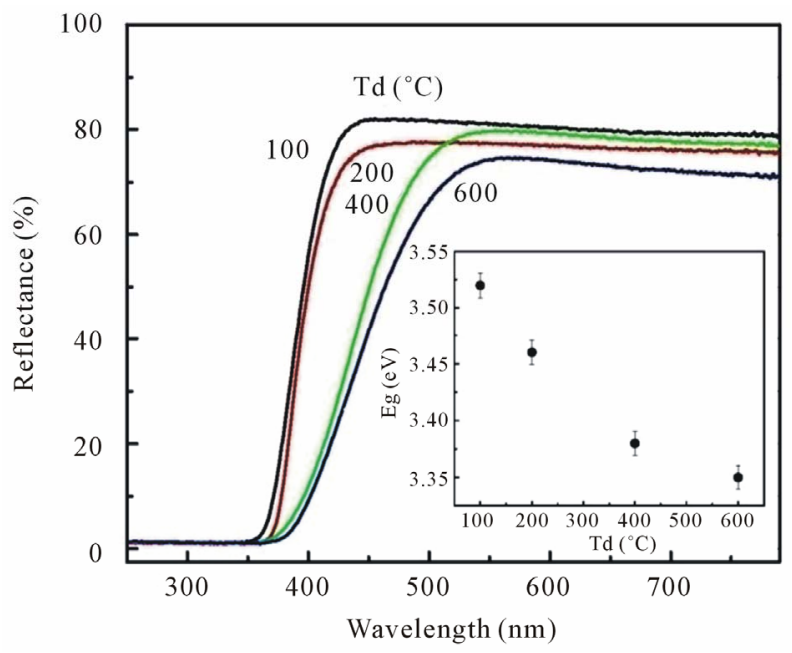

Figure 4. Diffuse reflectance spectra of $\mathrm{ZnO}$ nanoparticles synthesized with various dry temperatures. The inset shows the correlated optical gap of $\mathrm{ZnO}$ nanoparticles as a function of dry temperatures.

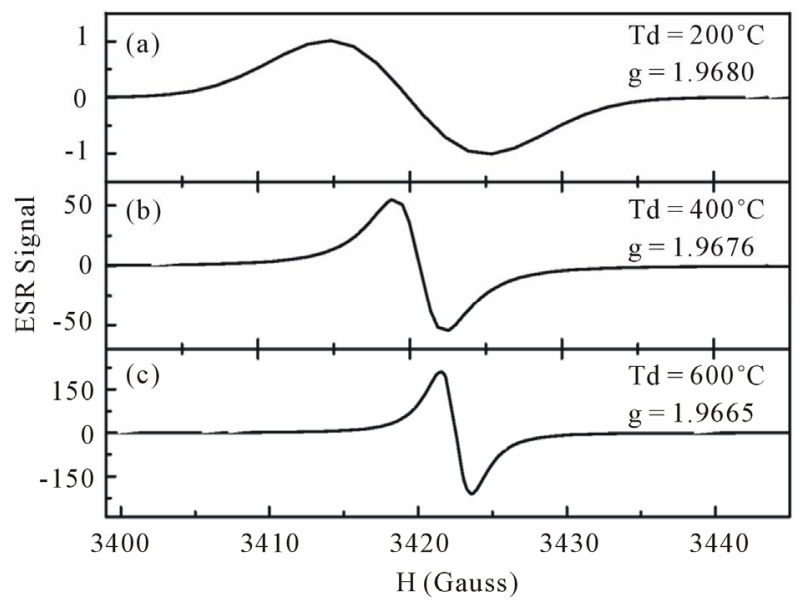

Figure 5. ESR spectra of $\mathrm{ZnO}$ nanoparticles dried at dry temperature of (a) $200^{\circ} \mathrm{C}$; (b) $400^{\circ} \mathrm{C}$; (c) $600^{\circ} \mathrm{C}$.

in higher temperatures (not shown in the figure). The resonance position for the spectrum corresponds to a $g$-value of 1.9680 for sample dried at $T d=200^{\circ} \mathrm{C}, 1.9676$ for $T d=400^{\circ} \mathrm{C}$ and 1.9665 for $T d=600^{\circ} \mathrm{C}$.

Although the origin of the signal with these $g$-values are still unclear, it has been demonstrated in the literature that the paramagnetic signal around $g$ value of 1.96 appears often owing to residual impurities in $\mathrm{ZnO}(\mathrm{F}, \mathrm{Cl}$, $\mathrm{Br}$ and $\mathrm{Al}, \mathrm{Ga}$, In; with the $g$-value being practically independent of the type of impurities) as well as intrinsic defects like oxygen vacancies or $\mathrm{Zn}$ interstitial [3,17-23]. Recently, Hoffmann et al. [22] conducted high-field ESR and ENDOR experiment at microwave frequencies of 95 and $275 \mathrm{GHz}$ on $\mathrm{ZnO}$ single crystal. Their results showed the ESR signal with $g$-values of $g / /=1.9569 \pm 0.00005$ and $g \perp=1.9552 \pm 0.00005$ ( $g$ parallel and perpendicular 
to the crystal c-axis), D1 and D2 centers. Using ENDOR technique, it was shown that hydrogen atom is involved in the D1 center. This result was in good agreement with the prediction of van de Walle [5]. However, the origin of D2 center so far remains unknown. In early publications $[3,17,19]$, it was shown that the $g$ value at 1.96 correlates with electron conductivity and originates from shallow donors. Kasai [3] and Hausmann et al. [18] argued that this line are related to the oxygen vacancies, whereas the EPR signal with the $g$ values of $g / /=1.9945$ and $g \perp$ $=1.9960$ was attributed by Hausmann and Schallenberger [18] to the oxygen atom in the tetrahedral interstitial position. Several authors [21,24-27], however associated the EPR signal at $g / /=1.9945$ and $g \perp=$ 1.9960 to an oxygen vacancy $\mathrm{V}^{+}$charge states, that are observed only in irradiated $\mathrm{ZnO}$ crystals under illumination and stable up to $400^{\circ} \mathrm{C}-500^{\circ} \mathrm{C}$ annealing.

By comparing our results with the results obtained in the literature above we believed that the $g$ values in our samples correlated with the oxygen sublattices that could be occupied with hydrogen atoms.

At lower dry temperature, almost all oxygen sublattices were occupied by hydrogen, made it difficult to detect the ESR signal. Therefore we could only detect weak ESR signal in samples dried with Td lower than $400^{\circ} \mathrm{C}$. At higher dry temperature the occupation number of hydrogen in the oxygen sublattices is reduced, leaving unoccupied and giving rise to an ESR signals. It is also shown that the integrated area increases essentially with an increase in dry temperatures. Our $g$-values are in line with the value reported previously from the $\mathrm{ZnO}$ single crystal, ceramic and powder $[3,17,18]$. These results showed that the ESR signal at $g \sim 1.96$ in our samples appears to its fullest intensity without irradiation or/and simultaneous illumination the samples.

To support this hypothesis we have annealed our samples in vacuum in order to diffuse hydrogen out of the samples. The results are displayed in Figure 5. It is seen that annealing the samples above $400^{\circ} \mathrm{C}$ had influenced the intensity of ESR signal as well as integrated area. Both are enhanced with increasing temperature annealing indicating that the number of paramagnetic center are increased with increasing annealing temperatures. We believe therefore our ESR results correspond to oxygen sublattices. The hydrogen atoms occupied the oxygen sublattice is considerable lower in our annealed samples than in the as-grown samples. Our ESR results are supported by the results obtained from infrared absorption spectra.

Infrared absorption measurements allow the study of hydrogen bonding configurations of as grown as well as annealed samples. Typical infrared absorption spectra of samples synthesized at different dry temperatures are displayed in Figure 6.

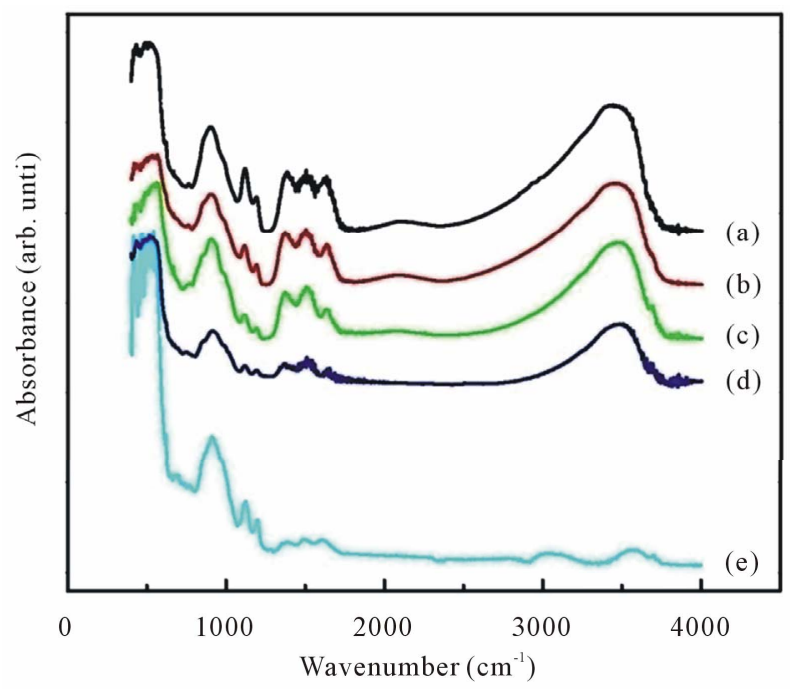

Figure 6. FTIR spectra of ZnO nanoparticles synthesized with various dry temperatures (curve (a)-(e)). Also shown the FTIR spectra of $\mathrm{ZnO}$ nanoparticles annealed with temperature annealing of $800^{\circ} \mathrm{C}$.

Also shown is the infrared spectrum for the sample dried at $100^{\circ} \mathrm{C}$ for $4 \mathrm{~h}$ and further annealed $800^{\circ} \mathrm{C}$ for $6 \mathrm{~h}$ (curve e). For all as-synthesized samples the strong absorption peaks in the range of $400-700 \mathrm{~cm}^{-1}$ could be attributed to $\mathrm{ZnO}$ stretching modes $[28,29]$. These stretching modes are indicative of successful synthesized of nanocrystalline $\mathrm{ZnO}$ particles as already confirmed by XRD and EDX studies. At the same time we can also observed an absorption peak around 1646, 1390 and $1121 \mathrm{~cm}^{-1}$ corresponds to $\mathrm{OH}$ bending mode, $\mathrm{C}-\mathrm{OH}$ in-off plane bending and $\mathrm{C}-\mathrm{OH}$ out-of-plane bending, respectively [30]. A broad band in the region $2900-3700$ $\mathrm{cm}^{-1}$ can be explained as overlaping of physically absorbed water, $\mathrm{O}-\mathrm{H}$ stretching modes and $\mathrm{C}-\mathrm{H}$ stretching modes. C-H local vibrational modes between 2800 and $3100 \mathrm{~cm}^{-1}$ have been observed in a number of semiconductor such as amorphous silicon carbon $(\mathrm{a}-\mathrm{SiC}: \mathrm{H})$, $\mathrm{GaAs}$, and $\mathrm{GaN}$ [31-33]. In these materials the local vibrational modes are assigned to symmetric and antisymmetric C-H stretching modes.

To obtain information more clearly on the local vibrational modes correspond to $\mathrm{OH}$ modes we focalized our analysis on the infrared absorption range of interest, namely in a wave number range of $2900-3700 \mathrm{~cm}^{-1}$. All spectra can be deconvoluted into two peaks around 2990 $\mathrm{cm}^{-1}$ attributed to $\mathrm{CH}$ stretching mode and $3400 \mathrm{~cm}^{-1}$ associated with physically absorbed water and $\mathrm{O}-\mathrm{H}$ stretching modes (dashed line in Figure 7).

An increase in dry temperature from $100^{\circ} \mathrm{C}$ to $600^{\circ} \mathrm{C}$ is seen to result in general decrease in the absorption peak height and in significant change of the spectral distribution. In particular a shift of absorption band to the higher wave number. Annealing the samples results in 
lowering of the peak height and a shift of peaks maximum towards higher wave number even further and the absorption peaks are diminished as the samples were annealed at temperature annealing of $800^{\circ} \mathrm{C}$ for $6 \mathrm{~h}$. The spectra of the samples dried at higher temperatures as well as the samples annealed at annealing temperatures lower than $800^{\circ} \mathrm{C}$ showed that $\mathrm{OH}$ band is still remained indicating that hydrogen remaining are chemically bonded to the oxygen in $\mathrm{ZnO}$ network. The total integrated absorption of the $\mathrm{CH}$ and $\mathrm{OH}$ stretching bands as a function of dry temperatures are plotted in Figure 8. The integrated absorption is evaluated by the sum of absorption

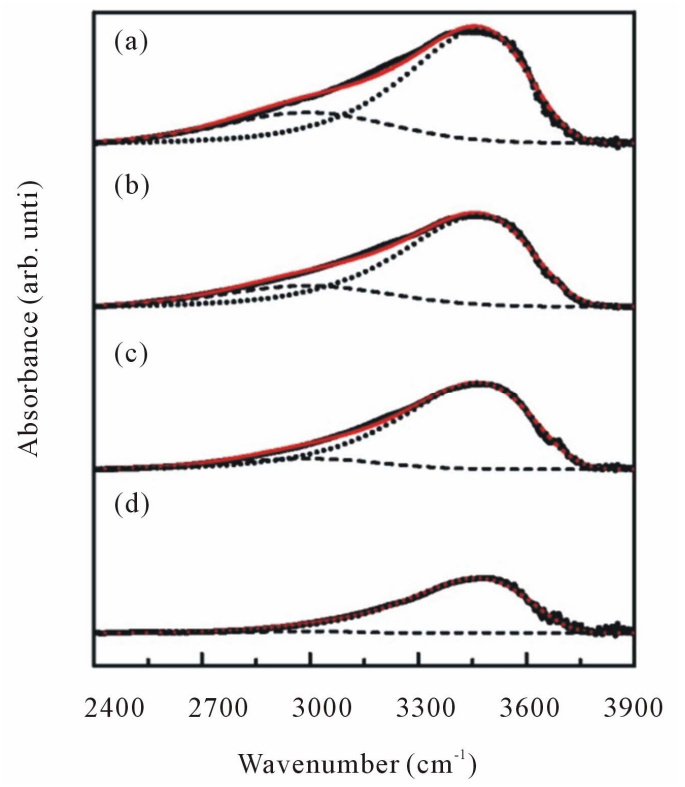

Figure 7. Representative deconvolution of $\mathrm{C}-\mathrm{H}$ stretching (dash line) and O-H stretching (dotted line) modes of infrared absorption band in the range of $2400-3900 \mathrm{~cm}^{-1}$ for $\mathrm{ZnO}$ nanoparticles synthesized with various dry temperatures.

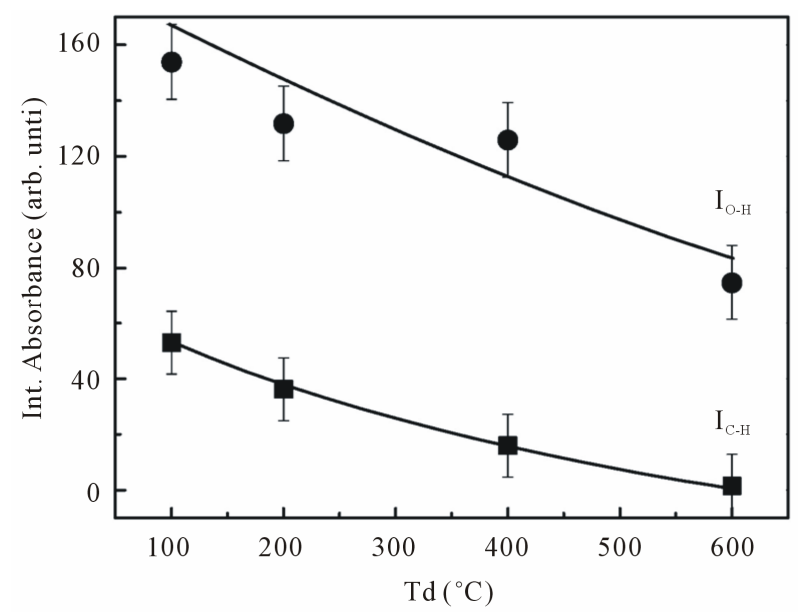

Figure 8. Integrated absorption of $\mathrm{C}-\mathrm{H}$ stretching mode at $2990 \mathrm{~cm}^{-1}$ and $\mathrm{O}-\mathrm{H}$ stretching mode at $3400 \mathrm{~cm}^{-1}$. areas of the fits. As one may observe in Figure 8, most of hydrogen atoms in our samples prefer to bond to oxygen atoms rather than carbon atoms. An increase in the dry temperature resulted in an overall decrease in the integrated absorption. This figure clearly showed that the dry temperature limits the incorporation of hydrogen atom bonded in the shape of $\mathrm{OH}$ as well as $\mathrm{CH}$ groups. This result is consistent with the ESR results describe previously. The influence of hydrogen incorporation on $g$-value in our ESR measurements shown in Figures 5 and 9 revealed that our ESR signal is dominated by paramagnetic centers due to oxygen sublattice.

\section{Summary}

In summary, a series of undoped nanocrystalline $\mathrm{ZnO}$ particles were synthesized using coprecipitation method at various dry temperatures. Some samples were annealed at annealing temperature up to $800^{\circ} \mathrm{C}$ for $6 \mathrm{~h}$. The properties of as-synthesized and annealed samples were characterized by various methods of measurements. XRD pattern for as-synthesized as well as annealed samples agree well with the standard XRD patern of $\mathrm{ZnO}$ with hexagonal wurzite structure. These results are consistent with the results obtained from EDX spectra. Moreover, all diffraction peaks revealed stronger peak intensities, indicating that the obtained $\mathrm{ZnO}$ particles have high crystallinity. The as-synthesized $\mathrm{ZnO}$ particles have an average of grain size $18-23 \mathrm{~nm}$, increased with increasing dry temperatures. UV-vis measurements showed the red shift in the optical absorption band with increasing average grain size. ESR spectra of our $\mathrm{ZnO}$ particles show the resonance of electron center with the $g$-value close to 1.96 . We believed that this resonance due to the oxygen sublattices which were occupied with hydrogen atoms. A decrease of the $g$-value and an increase of the oxygen sublattices were observed with decreasing hy-

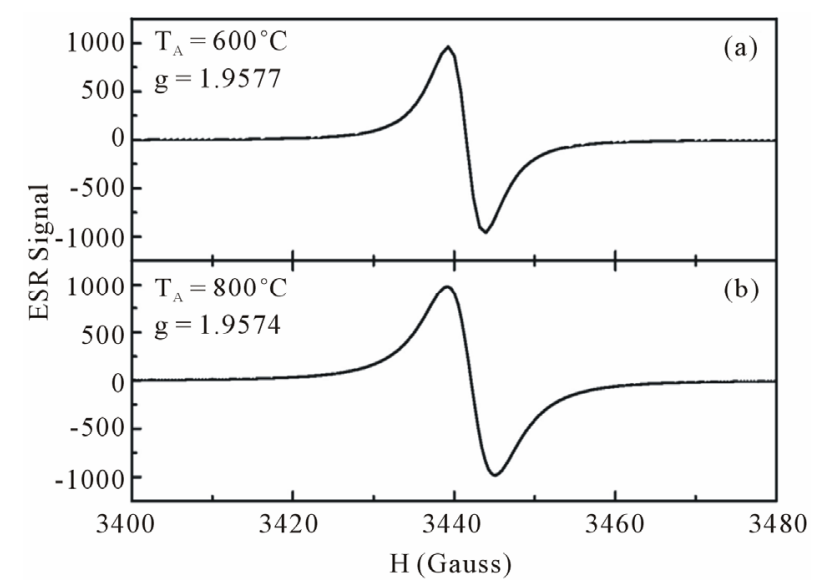

Figure 9. ESR spectra of $\mathrm{ZnO}$ nanoparticles annealed with temperature of anneling at $600^{\circ} \mathrm{C}$ (a) and $800^{\circ} \mathrm{C}$ (b). 
drogen incorporated in oxygen atoms, as can be seen from infrared absorption measurement.

\section{REFERENCES}

[1] C. W. Bunn, "The Lattice Dimensions of Zinc Oxide," Proceedings of the Physical Society, Vol. 47, No. 5, 1935, pp. 835-842. doi:10.1088/0959-5309/47/5/307

[2] F. Seitz and D. Turnbull, "Solid State Physics: Advances in Research and Application Vol 8," Academic Press Inc., New York, 1959.

[3] P. H. Kasai, "Electron Spin Resonance Studies of Donors and Acceptors in ZnO," Physical Review, Vol. 130, 1963, pp. 989-995. doi:10.1103/PhysRev.130.989

[4] U. Özgür, Y. I. Alivov, C. Liu, A. Teke, M. A. Reshchikov, S. Dogan, V. Avrutin, S. J. Cho and H. Morkoc, "A Comprehensive Review of $\mathrm{ZnO}$ Material and Devices," Journal of Applied Physics, Vol. 98, 2005, Article ID: 041301.

[5] C. G. Van de Walle, "Hydrogen as a Cause of Doping in Zinc Oxide," Physical Review Letters, Vol. 85, 2000, pp. 1012-1015. doi:10.1103/PhysRevLett.85.1012

[6] V. Ischenko, S. Polarz, D. Grote, V. Stavarache, K. Fink and M. Driess, "Zinc Oxide Nanoparticles with Defects," Advanced Functional Materials, Vol. 15, No. 12, 2003, pp. 1945-1954. doi:10.1002/adfm.200500087

[7] P. K. Sharma, A. C. Pandey, G. Zolnierkiewicz, N. Guskos and C. Rudowicz, "Relationship between Oxygen Defects and the Photoluminescence Property of $\mathrm{ZnO}$ Nanoparticles: A Spectroscopic View," Journal of Applied Physics, Vol. 106, No. 9, 2009, Article ID: 094314. doi: $10.1063 / 1.3256000$

[8] H. C. Huang and T. E. Hsieh, "Preparation and Characterization of Highly Tansparent UV-Curable ZnO-Acrylic Nanocomposites," Ceramics International, Vol. 36, No. 4, 2010, pp. 1245-1251. doi:10.1016/j.ceramint.2010.01.010

[9] K. V. Rajeswari and P. Gomathisankar, "Antibacterial and Photocatalytic Activities of Sonochemically Prepared $\mathrm{ZnO}$ and Ag-ZnO," Journal of Alloys and Compounds, Vol. 508, No. 2, 2010, pp. 587-591.

doi:10.1016/j.jallcom.2010.08.128

[10] Y. Hu and H. J. Chen, "Preparation and Characterization of Nanocrystalline $\mathrm{ZnO}$ Particles from a Hydrothermal Process," Journal of Nanoparticle Research, Vol. 10, No. 3, 2008, pp. 401-407. doi:10.1007/s11051-007-9264-0

[11] C. S. Lin, C. C. Hwang, W. H. Lee and W. Y. Tong, "Preparation of Zinc Oxyde ( $\mathrm{ZnO})$ Powder with Different Types of Morphology by a Combution Synthesis Method," Materials Science and Engineering: B, Vol. 140, No. 1-2, 2007, pp. 31-37. doi:10.1016/j.mseb.2007.03.023

[12] P. K. Giri, S. Bhattacharyya, D. K. Singh, R. Kesavamoorthy, B. K. Panigrahi and K. G. M. Nair, "Correlation between Microstructure and Optical Properties of $\mathrm{ZnO}$ Nanoparticles Synthesized by Ballmiling," Journal of Applied Physics, Vol. 102, No. 9, 2007, Article ID: 093515. doi:10.1063/1.2804012

[13] Gao, Z. Zhang, J. Fu, Y. Xu, J. Qi and D. Xue, "Room
Temperature Ferromagnetism of Pure $\mathrm{ZnO}$ Nanoparticles," Journal of Applied Physics, Vol. 105, No. 11, 2009, Article ID: 113928. doi:10.1063/1.3143103

[14] A. Thurber, K. M. Reddy, V. Shutthanandan, M. H. Engelhard, C. Wang, J. Hays and A. Punnoose, "Ferromagnetism in Chemically Synthesized $\mathrm{CeO}_{2}$ Nanoparticles by Ni Doping," Physical Review B, Vol. 76, 2007, Article ID: 165206. doi:10.1103/PhysRevB.76.165206

[15] M. Naeem, S. K. Hasanain and A. Mumtaz, "Electrical Transport and Optical Studies of Ferromagnetic Cobalt Doped ZnO Nanoparticles Exhibiting a Metal-Insulator Transition," Journal of Physics: Condensed Matter, Vol. 20, No. 2, 2008, Article ID: 025210. doi:10.1088/0953-8984/20/02/025210

[16] L. Irimpan, V. P. N. Nampoori, P. Radhakrishnan, B. Krishnan and A. Deepthy, "Size-Dependent Enhancement of Nonlinear Optical Properties in Nanocolloids of ZnO," Journal of Applied Physics, Vol. 103, No. 3, 2008, Article ID: 033105. doi: 10.1063/1.2838178

[17] K. M. Sancier, "ESR Investigation of Photodamage to Zinc Oxide Powders," Surface Science, Vol. 21, No. 1, 1970, pp. 1-11. doi:10.1016/0039-6028(70)90059-2

[18] A. Hausmann and B. Schallenberger, "Interstitial Oxygen in Zinc Oxide Single Crystals," Zeitschrift fur Physik, Vol. 31, 1978, pp. 269-273.

[19] M. Schulz, "ESR Experiments on Ga Donors in $\mathrm{ZnO}$ Crystals," Phys Status Solidi, Vol. 27, No. 1, 1975, pp. K5-K8. doi:10.1002/pssa.2210270140

[20] W. E. Carlos, E. R. Glaser and D. C. Look, "Magnetic Resonance Studies of ZnO," Physical B, Vol. 308, 2001, pp. 976-979. doi:10.1016/S0921-4526(01)00850-X

[21] V. A. Nikitenko, K. E. Tarkpea, I. V. Pykanov and S. G. Stoyukhin, "EPR and Thermoluminescence in $\mathrm{ZnO}$ Single Crystals with Ionic Vacancies," Journal of Applied Spectroscopy, Vol. 68, No. 3, 2001, pp. 502-507. doi:10.1023/A:1011975028398

[22] D. M. Hoffmann, A. Hofstaetter, F. Leiter, H. Zhou, F. Henecker and B. K. Meyer, "Hydrogen: A Relevant Shallow Donor in Zinc Oxide," Physical Review Letters, Vol. 88, 2002, Article ID: 045504. doi:10.1103/PhysRevLett.88.045504

[23] H. Zhou, A. Hofstaetter, D. M. Hofmann and B. K. Meyer, "Magnetic Resonance Studies on ZnO Nanocrystals," Microelectronic Engineering, Vol. 66, No. 1-4, 2003, pp. 59-64. doi:10.1016/S0167-9317(03)00025-X

[24] J. M. Smith and W. E. Vehse, "ESR of Electron Irradiated ZnO Confirmation of $\mathrm{F}^{+}$Center," Physics Letters A, Vol. 31, No. 3, 1970, pp. 147-148. doi:10.1016/0375-9601(70)90199-4

[25] J. M. Meese and D. R. Locker, "Oxygen Displacement Energy in ZnO," Solid State Communications, Vol. 11, No. 11, 1972, pp. 1547-1550. doi:10.1016/0038-1098(72)90517-0

[26] L. S. Vlasenko and G. D. Watkins, "Optical Detection of Electron Paramagnetic Resonance in Room-Temperature Electron-Irradiated ZnO," Physical Review B, Vol. 71, 2005, Article ID: 125210 . doi:10.1103/PhysRevB.71.125210 
[27] L. S. Vlasenko, "Magnetic Resonance Studies of Intrinsic Defects in ZnO: Oxygen Vacancy," Applied Magnetic Resonance, Vol. 39, No. 1-2, 2010, pp. 103-111. doi:10.1007/s00723-010-0140-1

[28] W. Xie and X. Huang, "Synthesis of Biodiesel from Soybean Oil Using Heterogeneous KF/ZnO Catalyst," Catalysis Letters, Vol. 107, No. 1-2, 2006, pp. 53-59. doi:10.1007/s10562-005-9731-0

[29] A. Hernández, L. Maya, E. Sánchez-Mora and E. M. Sánchez, "Sol-Gel Synthesis, Characterization and Photocatalytic Activity of Mixed Oxide $\mathrm{ZnO}-\mathrm{Fe}_{2} \mathrm{O}_{3}$," Journal of Sol-Gel Science and Technology, Vol. 42, No. 1, 2007, pp. 71-78. doi:10.1007/s10971-006-1521-7

[30] J. Das, I. R. Evans and D. Khushalani, "Zinc Glycolate: A Precursor to ZnO," Inorganic Chemistry, Vol. 48, No. 8,
2009, pp. 3508-3510. doi:10.1021/ic900067w

[31] R. Saleh, M. Munisa and W. Beyer, "Infrared Absorption in a-SiC:H Films Prepared by DC Sputtering," Thin Solid Films, Vol. 426, No. 1-2, 2003, pp. 117-123. doi:10.1016/S0040-6090(03)00003-8

[32] D. M. Joseph, R. Balagopal, R. F. Hicks, L. P. Sadwick and K. L. Wang, "Observation of Carbon Incorporation during Gallium Arsenide Growth by Molecular Beam Epitaxy," Applied Physics Letters, Vol. 53, No. 22, 1988, pp. 2203-2204. doi:10.1063/1.100281

[33] M. O. Manasreh, J. M. Baranowski, K. Pakula, H. X. Jiang and J. Lin, "Localized Vibrational Mode of CarbonHydrogen Complexes in GaN," Applied Physics Letters, Vol. 75, No. 5, 1999, pp. 659-661. doi:10.1063/1.124473 